\title{
Decreasing mortality and changes in treatment patterns in patients with acromegaly from a nationwide study
}

\section{Daniela Esposito', Oskar Ragnarsson', Daniel Granfeldt ${ }^{2}$, Tom Marlow², Gudmundur Johannsson ${ }^{1}$ and Daniel S Olsson ${ }^{1}$}

${ }^{1}$ Department of Endocrinology, Institute of Medicine, Sahlgrenska Academy, University of Gothenburg and Sahlgrenska University Hospital and ${ }^{2}$ Nordic Health Economics, Gothenburg, Sweden

Correspondence should be addressed to D S Olsson Email daniel.olsson@gu.se

The authors apologise for errors that have appeared in the article by Espito et al. published in the May 2018 issue of the European Journal of Endocrinology (vol 178, iss 5, pages 459-469).

They state that after publishing the paper, the collaboration between the research parties was continued to further understand the excess mortality that was found among patients with acromegaly in Sweden between 1987 and 2013. During this process, the authors discovered that all standardized mortality ratios (SMRs) in the article were systematically overestimated. The correct SMRs are summarised in Table 6 below.

The authors have suggested the following changes to the various sections in their article.

\section{Changes to the Abstract}

Results: Overall SMR was 1.33 (95\% CI: 1.17-1.52) with 232 observed and 174 expected deaths. Mortality was mainly related to circulatory diseases (SMR: 1.34 (95\% CI: 1.08-1.64)), including cerebrovascular disease (1.63, 1.06-2.41). Mortality was only increased during the first time period, suggesting a reduction in mortality over time.

Conclusions: Excess mortality was found in this nationwide cohort of patients with acromegaly, mainly related to circulatory diseases.

\section{Changes to the Results section}

The corrected Fig. 1, Tables 3 and 5 are printed in full below. Supplementary Table 3 (see section on supplementary data given at the end of this article) has also been revised and is available with the online version of this Erratum article.

A summary of the correct SMRs is presented in Table 6.


\section{Figure 1}

Mortality in patients with acromegaly in Sweden followed between 1987 and 2013. Overall mortality in the entire cohort of patients (A), men (B) and women (C) with acromegaly. *** $P<0.001$. https://eje.bioscientifica.com https://doi.org/10.1530/EJE-18-0015e (c) 2019 European Society of Endocrinology Printed in Great Britain
Published by Bioscientifica Ltd. 


\section{Changes to the Discussion section}

Page 464: Mortality due to ischemic heart disease and malignancy was not increased.

Page 465: We did not observe an excess mortality from malignancies, which is in line with a Finnish study including 333 patients followed for 20 years.

Page 465: The mortality was only increased during the first time period (1987-1995) suggesting decreasing mortality over time.

Page 467: In conclusion, excess mortality was found in this nationwide cohort of unselected patients with acromegaly, mainly related to circulatory diseases.

The authors once again apologise for these errors.

Table 3 Standardized mortality ratio in patients with acromegaly in Sweden between 1987 and 2013.

\section{Mortality}

Overall mortality

Acromegalic patients $(n=1089)$

Men $(n=509)$

Women $(n=580)$

Cause-specific mortality (ICD chapters)

ICD-10 chapter 1, infectious disease

Men $(n=509)$

Women $(n=580)$

ICD-10 chapter 9, circulatory disease

Men $(n=509)$

Women $(n=580)$

ICD-10 chapter 10, respiratory disease

Men $(n=509)$

Women $(n=580)$

Cause-specific mortality (specific causes)

Ischemic heart disease

Men $(n=509)$

Women $(n=580)$

Cerebrovascular disease*

Men $(n=509)$

Women $(n=580)$

Malignant neoplasms

Men $(n=509)$

Women $(n=580)$

Malignant neoplasm of brain

Overall mortality in subgroups

All patients treated with surgery $(n=700)$

Men $(n=340)$

Women $(n=360)$

Patients treated with surgery alone $(n=584)$

Men $(n=290)$

Women $(n=294)$

All patients treated with radiotherapy $(n=144)$

Men $(n=67)$

Women $(n=77)$

Patients treated with radiotherapy alone $(n=28)$

Men $(n=17)$

Women $(n=11)$

Patients treated with both surgery and radiotherapy $(n=116)$

Men $(n=50)$

Women $(n=66)$

Patients treated without both surgery and radiotherapy $(n=361)$

Men $(n=152)$

Women $(n=209)$
No. observed

No. expected

232

107

125

6

2

4

93

38

55

14

5

9

36

15

21

25

9

16

49

27

22

3

00

46

54

91

41

50

18

10

8

9

5

4

9

5

4

123

56

67

No. expected

174.1

80.7

93.5

2.7

1.2

1.5

69.3

32.2

37.1

10.7

4.7

6.0

33.2

17.8

15.5

15.3

5.8

9.5

50.6

24.1

26.5

1.4

85.5

42.0

43.5

75.6

37.0

38.6

8.9

4.8

4.1

3.2

2.2

1.0

5.7

2.6

3.1

80.1

34.2

45.9

SMR $(95 \% \mathrm{Cl})$

$1.33(1.17-1.52)$

$1.33(1.09-1.60)$

$1.34(1.11-1.59)$

$2.20(0.81-4.79)$

NC

$2.60(0.71-6.66)$

$1.34(1.08-1.64)$

$1.18(0.84-1.62)$

$1.48(1.12-1.93)$

$1.31(0.71-2.19)$

$1.06(0.35-2.48)$

$1.49(0.68-2.83)$

$1.08(0.76-1.50)$

$0.85(0.47-1.40)$

$1.36(0.84-2.07)$

$1.63(1.06-2.41)$

$1.54(0.71-2.93)$

$1.69(0.96-2.74)$

$0.97(0.72-1.28)$

$1.12(0.74-1.63)$

$0.83(0.52-1.26)$

$2.16(0.45-6.32)$

$1.17(0.95-1.42)$

$1.10(0.80-1.46)$

$1.24(0.93-1.62)$

$1.20(0.97-1.48)$

$1.11(0.80-1.50)$

$1.30(0.96-1.71)$

$2.02(1.20-3.20)$

$2.07(0.99-3.80)$

$1.97(0.85-3.88)$

$2.82(1.29-5.34)$

$2.25(0.73-5.26)$

$4.09(1.12-10.5)$

$1.59(0.73-3.01)$

$1.93(0.63-4.50)$

$1.30(0.35-3.32)$

$1.54(1.28-1.83)$

$1.64(1.24-2.12)$

$1.46(1.13-1.86)$
P Value

$<0.0001$

0.006

0.002

0.12

NC

0.14

0.008

0.35

0.007

0.39

0.99

0.31

0.68

0.61

0.21

0.028

0.27

0.066

0.90

0.61

0.44

0.33

0.13

0.58

0.14

0.093

0.55

0.089

0.009

0.053

0.11

0.011

0.15

0.035

0.24

0.24

0.74

$<0.0001$

0.0008

0.004

*No deaths caused by cerebrovascular disease were observed in patients treated with radiotherapy. $\mathrm{Cl}$, confidence interval; NC, not calculated; SMR, standardized mortality ratio. 
Table 5 Time trends in mortality in patients with acromegaly in Sweden followed between 1987 and 2013.

\begin{tabular}{|c|c|c|c|c|}
\hline Mortality & No. observed & No. expected & SMR $(95 \% \mathrm{Cl})$ & P Value \\
\hline \multicolumn{5}{|c|}{ Overall mortality } \\
\hline 1987-1995 & 139 & 99.6 & $1.40(1.17-1.65)$ & 0.0002 \\
\hline 1996-2004 & 73 & 60.1 & $1.21(0.95-1.53)$ & 0.12 \\
\hline 2005-2013 & 20 & 14.4 & $1.40(0.85-2.15)$ & 0.18 \\
\hline \multicolumn{5}{|l|}{ Men } \\
\hline 1987-1995 & 59 & 46.2 & $1.28(0.97-1.65)$ & 0.078 \\
\hline 1996-2004 & 37 & 28.0 & $1.32(0.93-1.83)$ & 0.12 \\
\hline 2005-2013 & 11 & 6.5 & $1.69(0.84-3.02)$ & 0.13 \\
\hline \multicolumn{5}{|l|}{ Women } \\
\hline 1987-1995 & 80 & 53.4 & $1.50(1.19-1.86)$ & 0.0008 \\
\hline 1996-2004 & 36 & 32.2 & $1.12(0.78-1.55)$ & 0.55 \\
\hline 2005-2013 & 9 & 7.8 & $1.15(0.53-2.18)$ & 0.77 \\
\hline \multicolumn{5}{|c|}{ Patients treated with surgery alone } \\
\hline 1987-1995 & 63 & 44.5 & $1.42(1.09-1.81)$ & 0.010 \\
\hline 1996-2004 & 26 & 25.1 & $1.03(0.68-1.52)$ & 0.92 \\
\hline 2005-2013 & 2 & 5.9 & $\mathrm{NC}$ & NC \\
\hline \multicolumn{5}{|c|}{ Patients treated with radiotherapy alone } \\
\hline 1987-1995 & 4 & 1.5 & $2.60(0.71-6.65)$ & 0.14 \\
\hline 1996-2004 & 4 & 0.9 & $4.30(1.17-11.02)$ & 0.030 \\
\hline 2005-2013 & 1 & 0.7 & NC & NC \\
\hline \multicolumn{5}{|c|}{ Patients treated with both surgery and radiotherapy } \\
\hline 1987-1995 & 4 & 1.3 & $3.07(0.84-7.87)$ & 0.086 \\
\hline 1996-2004 & 3 & 3.7 & $0.80(0.17-2.35)$ & 0.97 \\
\hline 2005-2013 & 2 & 0.6 & $\mathrm{NC}$ & NC \\
\hline \multicolumn{5}{|c|}{ Patients treated without both surgery and radiotherapy } \\
\hline 1987-1995 & 68 & 46.9 & $1.45(1.13-1.84)$ & 0.0044 \\
\hline 1996-2004 & 40 & 27.3 & $1.46(1.05-1.99)$ & 0.027 \\
\hline 2005-2013 & 15 & 5.9 & $2.53(1.41-4.16)$ & 0.003 \\
\hline
\end{tabular}

Table 6 Standardized mortality ratio in patients with acromegaly in Sweden between 1987 and 2013.

\begin{tabular}{l}
\hline Mortality \\
\hline Overall mortality \\
Acromegalic patients $(n=1089)$ \\
Men $(n=509)$ \\
Women $(n=580)$ \\
Cause-specific mortality (ICD chapters) \\
ICD-10 chapter 9, circulatory disease \\
ICD-10 chapter 10, respiratory disease \\
Cause-specific mortality (specific causes) \\
Ischemic heart disease \\
Cerebrovascular disease \\
Malignant neoplasms
\end{tabular}

\begin{tabular}{c}
\hline No. observed \\
\hline 232 \\
107 \\
125 \\
93 \\
14 \\
36 \\
25 \\
49 \\
\hline
\end{tabular}

\begin{tabular}{c}
\hline No. expected \\
\hline 174.1 \\
80.7 \\
93.5 \\
69.3 \\
10.7 \\
33.2 \\
15.3 \\
50.6
\end{tabular}

\begin{tabular}{c}
\hline SMR $(95 \% \mathrm{CI})$ \\
\hline $1.33(1.17-1.52)$ \\
$1.33(1.09-1.60)$ \\
$1.34(1.11-1.59)$ \\
$1.34(1.08-1.64)$ \\
$1.31(0.71-2.19)$ \\
$1.08(0.76-1.50)$ \\
$1.63(1.06-2.41)$ \\
$0.97(0.72-1.28)$ \\
\hline
\end{tabular}

\begin{tabular}{c}
\hline \multicolumn{1}{c}{$\boldsymbol{P}$ Value } \\
\hline$<0.0001$ \\
0.0058 \\
0.0021 \\
\\
0.0077 \\
0.39 \\
\\
0.68 \\
0.028 \\
0.90
\end{tabular}

$\mathrm{Cl}$, confidence interval; SMR, standardized mortality ratio.

Supplementary data

This is linked to the online version of this erratum at https://doi.org/10.1530/EJE-18-0015e. 